\title{
A SHARP ESTIMATE ON THE NORM OF THE MARTINGALE TRANSFORM
}

\author{
JANINE WITTWER
}

\section{Introduction}

The boundedness of singular integral operators in $L^{2}(w)$ for $w \in A_{2}$ has been known for a long time, by the Hunt-Muckenhaupt-Wheeden Theorem. What is as of yet unknown is the sharp bound of these operators in terms of the $A_{2}$ norm of $w$. In his thesis, S. Buckley proved that the Hardy-Littlewood maximal operator is bounded linearly in $\|w\|_{A_{2}}$, the square function operator bound is no more than $\|w\|_{A_{2}}^{3 / 2}$ and that the Hilbert transform norm is no worse than quadratic. It is easily seen that each of these operators can not have a better bound than linear in $\|w\|_{A_{2}}$ (just look at power weights). In this paper, following the methods of [6], we show that the bound for the martingale transform, which is a dyadic analog of singular integral operators, is linear. We also give a simple proof that the dyadic square function is bounded linearly.

\section{Notation}

In what follows, $h_{I}$ will denote the normalized Haar function for the dyadic interval $I$, i.e., $h_{I}=\frac{\chi_{I_{l}}-\chi_{I_{r}}}{\sqrt{|I|}}$, where $I_{l}, I_{r}$ denote the left and right children of $I$ respectively. The weight $w$ and its inverse $w^{-1}$ will be dyadic $A_{2}$ weights on $[0,1]$. $w$ will be normalized to have $\int_{[0,1]} w(x)=1$. Let $(f)_{I}$ denote $\frac{1}{|I|} \int_{I} f(x) d x$ for $I$ a dyadic interval. Sometimes the parentheses are omitted when it is clear which function we are averaging. $w_{I}^{-1}$ will denote $\frac{1}{|I|} \int_{I} \frac{1}{w(x)} d x$. Let $\mu(I)=w_{I} w_{I}^{-1}$ and $\|w\|_{A_{2}}=\sup _{I \in D} \mu(I)$.

We will write $w$ in the form $w(x)=\prod\left(1+c_{I} h_{I}\right)$ where $c_{I}=\frac{w_{I_{l}}-w_{I_{r}}}{2 w_{I}} \sqrt{|I|}$. Similarly, we define $d_{I}=\frac{w_{I_{l}}^{-1}-w_{I_{r}}^{-1}}{2 w_{I}^{-1}} \sqrt{|I|}$.

$\langle,\rangle_{\mu}$ will denote the inner product in $L^{2}(d \mu)$. If the subscript is omitted, the measure is $d x$.

The family of operators which we are concerned with are the martingale transforms

$$
T_{r} f=\sum_{I \in D[0,1]} r(I)\left\langle f, h_{I}\right\rangle h_{I}
$$

where $r(I)$ assumes the values +1 and -1 only.

Received March 2, 1999. Revised July 9, 1999. 


\section{The main theorem}

Theorem 3.1.

$$
\left\|T_{r} f\right\|_{L^{2}(w)} \leq c\|w\|_{A_{2}}\|f\|_{L^{2}(w)},
$$

for $w \in A_{2}$ and $f \in L^{2}(w)$.

We will prove this Theorem in section 5 .

\section{Useful lemmas and theorems}

The following theorem can be found in [6], Section 2 .

Theorem 4.1. Let $\alpha_{I} \geq 0$. Then

$$
\sum_{I \in D[0,1]}\left(f w^{1 / 2}\right)_{I}^{2} \alpha_{I} \leq 4 c\|f\|_{2}^{2}
$$

for all $f \in L^{2}(d x)$ iff

$$
\frac{1}{|J|} \sum_{I \subset J}\left(w_{I}\right)^{2} \alpha_{I} \leq c w_{J}
$$

for all $J \in D[0,1]$.

Theorem 4.2. Let $\alpha_{I} \geq 0$. If

$$
\frac{1}{|J|} \int_{J}\left(\sum_{I \subset J} \alpha_{I} w_{I} \chi_{I}(x)\right)^{2} w^{-1} d x \leq c_{1} w_{J}
$$

for all $J \in D$ and

$$
\frac{1}{|J|} \int_{J}\left(\sum_{I \subset J} \alpha_{I} w_{I}^{-1} \chi_{I}(x)\right)^{2} w d x \leq c_{1} w_{J}^{-1}
$$

for all $J \in D$, then

$$
\sum_{I \in D} \alpha_{I}\left(f w^{1 / 2}\right)_{I}\left(g w^{-1 / 2}\right)_{I}|I| \leq c_{2}\|f\|_{L^{2}}\|g\|_{L^{2}},
$$

for all $f, g \in L^{2}(d x)$, where $c_{2} \leq c \sqrt{c_{1}}$ and $c$ is an absolute constant.

Proof. From [6], section 4, we know that if

$$
\frac{1}{|J|} \int_{J}\left(\sum_{I \subset J} \alpha_{I} w_{I} \chi_{I}(x)\right)^{2} v(x) d x \leq w_{J}
$$

for all $J \in D$, then

$$
\sum_{I \in D_{1}} \alpha_{I}\left(f w^{1 / 2}\right)_{I}\left(g v^{1 / 2}\right)_{I}|I| \leq c\left(\|f\|_{L^{2}}^{2}+\|g\|_{L^{2}}^{2}\right),
$$

where $D_{1}=\left\{I \in D: \frac{\left(f w^{1 / 2}\right)_{I}^{2}}{w_{I}} \geq \frac{\left(g v^{1 / 2}\right)_{I}^{2}}{v_{I}}\right\}$.

To prove Theorem 4.2, we use this theorem with $v=\frac{w^{-1}}{c_{1}}$. Then, since in the conclusion $v$ appears with a square root only, the bound for the conclusion 
would be of the order $\sqrt{c_{1}}$. Furthermore, imposing that the condition be fulfilled for both $w$ and $w^{-1}$, we can sum over all dyadic intervals, since for all of them either

$$
\frac{\left(f w^{1 / 2}\right)_{I}^{2}}{w_{I}} \geq \frac{\left(g\left(w^{-1}\right)^{1 / 2}\right)_{I}^{2}}{w_{I}^{-1}}
$$

or

$$
\frac{\left(f w^{1 / 2}\right)_{I}^{2}}{w_{I}} \leq \frac{\left(g\left(w^{-1}\right)^{1 / 2}\right)_{I}^{2}}{w_{I}^{-1}}
$$

is true. Finally, letting $g^{*}=\left(\frac{\|g\|_{L^{2}}}{\|f\|_{L^{2}}}\right)^{1 / 2} g$ and $f^{*}=\left(\frac{\|g\|_{L^{2}}}{\|f\|_{L^{2}}}\right)^{-1 / 2} f$, we get

$$
\sum_{I \in D_{1}} \alpha_{I}\left(f^{*} w^{1 / 2}\right)_{I}\left(g^{*}\left(w^{-1}\right)^{1 / 2}\right)_{I}|I| \leq 2 c\left(\left\|f^{*}\right\|_{L^{2}}\left\|g^{*}\right\|_{L^{2}}\right)
$$

since this normalization does not change the right hand side at all.

The following estimate is not used in the proof of the main theorem, but may be interesting in its own right. It gives the (unweighted) carleson norm of the sequence $\left\{c_{I}\right\}$. See [7], theorem 4.1 .

Lemma 4.3. For $w \in A_{2}$ where $w(x)=C \prod\left(1+c_{I} h_{I}\right)$, the following is true:

$$
\sum_{I \subset J} c_{I}^{2} \leq 2 \log \left(\|w\|_{A_{2}}\right)|J|
$$

and this estimate is sharp.

Proof. By the definition of $w$,

$$
\begin{aligned}
\left(1-c_{I}^{2} /|I|\right)=1-\left(\frac{w_{I_{l}}-w_{I_{r}}}{2 w_{I}}\right)^{2} & = \\
& \frac{4\left(1 / 2\left(w_{I_{l}}+w_{I_{r}}\right)\right)^{2}-\left(w_{I_{l}}-w_{I_{r}}\right)^{2}}{\left(2 w_{I}\right)^{2}}=\frac{w_{I_{l}} w_{I_{r}}}{\left(w_{I}\right)^{2}}
\end{aligned}
$$

and therefore

$$
\frac{w_{I_{l}} w_{I_{r}}}{\left(w_{I}\right)^{2}} \leq e^{-c_{I}^{2} /|I|}
$$

Taking square roots, this becomes $w_{I} \geq e^{c_{I}^{2} / 2|I|}\left(w_{I_{l}} w_{I_{r}}\right)^{1 / 2}$.

Since $\left(w_{I}^{-1}\right)^{2} \geq\left(w_{I_{l}}^{-1} w_{I_{r}}^{-1}\right)$, we can multiply these two equations to get

$$
\mu(I) \geq e^{c_{I}^{2} / 2|I|}\left(\mu\left(I_{l}\right) \mu\left(I_{r}\right)\right)^{1 / 2} .
$$

Now use the same inequality to replace $\mu\left(I_{l}\right)$ and $\mu\left(I_{r}\right)$, and repeat this process. After $n$ steps we get

$$
\mu(I) \geq \exp \left(\sum_{K \subset I,|K| \geq 2^{-n}|I|} \frac{c_{K}^{2}|K|}{2|K||I|}\right)\left(\prod_{|K|=2^{-n}|I|} \mu(K)\right)^{2^{-n}}
$$


(The $|K| /|I|$ in the exp comes from the repeated square roots that we took.) Realizing that $\mu(K) \geq 1$ always, we get

$$
\mu(I) \geq \exp \left(\sum_{K \subset I,|K| \geq 2^{-n}|I|} \frac{c_{K}^{2}}{2|I|}\right) .
$$

Taking the limit as $n \rightarrow \infty$, and taking logarithms, we get the lemma.

To see that the bound is indeed sharp, consider $w=\exp \left(\sum_{i \in \mathbb{N}} b_{i} \Phi_{i}\right)$ where $\Phi_{i}$ are Rademacher functions. Then $\sum_{I \subset J} c_{I}^{2}=\sum_{i \geq j} \tanh ^{2}\left(b_{i}\right)|J|$, while $\|w\|_{A_{2}}=$ $\prod_{i \in \mathbb{N}}\left(1+\sinh ^{2}\left(b_{i}\right)\right)$.

Theorem 4.4. Let $S f$ be the dyadic square function of $f$. Then

$$
\|S f\|_{L^{2}(w)} \leq c\|w\|_{A_{2}}\|f\|_{L^{2}(w)},
$$

and this estimate is sharp.

Note. This estimate has also been proven, using a different method, in [5].

Proof. Let

$$
f_{I}=\frac{h_{I}}{\left(w_{I}\right)^{1 / 2}}
$$

and

$$
g_{I}=h_{I}\left(w_{I}\right)^{1 / 2} w^{-1} .
$$

Then $\left\langle f_{I}, g_{J}\right\rangle_{w}=1$ if $I=J$ and 0 otherwise. Let $f \in L^{2}(w)$.

$$
\|S f\|_{L^{2}(w)}=\left(\sum_{I \in D}\left\langle f, h_{I}\right\rangle^{2} w_{I}\right)^{1 / 2}=\left(\sum_{I \in D}\left\langle f, g_{I}\right\rangle_{w}^{2}\right)^{1 / 2}
$$

In that notation, the theorem to prove becomes

$$
\sum_{I \in D}\left\langle f, g_{I}\right\rangle_{w}^{2} \leq\|w\|_{A_{2}}^{2}\|f\|_{L^{2}(w)}^{2} .
$$

We will bound this by first showing that

$$
\sum_{I \in D}\left\langle f, f_{I}\right\rangle_{w}^{2} \leq\|w\|_{A_{2}}\|f\|_{L^{2}(w)}^{2},
$$

from which the desired inequality can be extracted by algebraic manipulations.

By the theorem on the lower bound of the square function in [3] we know the following:

$$
\|f\|_{L^{2}(w)} \leq c\|w\|_{A_{2}}{ }^{1 / 2}\left(\sum_{I \in D}\left\langle f, g_{I}\right\rangle_{w}^{2}\right)^{1 / 2}
$$


This allows us to compute the norm of the sequence valued operator $(J g)_{I}=$ $\left\langle g, f_{I}\right\rangle_{w}$ from $L^{2}(w)$ to $l^{2}$ :

$$
\begin{aligned}
& \sup _{\|g\|_{L^{2}(w)}=1} \sup _{\left\|\left\{k_{I}\right\}\right\|_{l^{2}}=1} \sum_{I \in D} k_{I} \int g f_{I} w= \\
& \sup _{\|g\|_{L^{2}(w)}=1} \sup _{\left\|\left\{k_{I}\right\}\right\|_{l^{2}=1}} \int g \sum_{I \in D} k_{I} f_{I} w \leq
\end{aligned}
$$

by Cauchy-Schwarz, and the above inequality,

$$
\begin{aligned}
\sup _{\left\|\left\{k_{I}\right\}\right\|_{l^{2}}=1} \sup _{\|g\|_{L^{2}(w)}=1}\left\|\sum_{I \in D} k_{I} f_{I}(x)\right\|_{L^{2}(w)}\|g\|_{L^{2}(w)} \leq \\
\|w\|_{A_{2}}^{1 / 2} \sup _{\left\|\left\{k_{I}\right\}\right\|_{l^{2}=1}}\left[\sum_{J \in D}\left\langle\left(\sum_{I \in D} k_{I} f_{I}\right), g_{J}\right\rangle_{w}^{2}\right]^{1 / 2}= \\
\|w\|_{A_{2}}^{1 / 2} \sup _{\left\|\left\{k_{I}\right\}\right\|_{l^{2}}=1}\left(\sum \sum_{I \in D} k_{I}^{2}\right)^{1 / 2}=\|w\|_{A_{2}}^{1 / 2}
\end{aligned}
$$

This means that

$$
\sum_{I \in D}\left\langle g, f_{I}\right\rangle_{w}^{2} \leq\|w\|_{A_{2}}\|g\|_{L^{2}(w)}^{2},
$$

for every weight in $A_{2}$. In particular, this is true for the weight $w^{-1}$ :

$$
\sum_{I \in D}\left\langle g, \frac{h_{I}}{\left(w_{I}^{-1}\right)^{1 / 2}}\right\rangle_{w^{-1}}^{2} \leq\|w\|_{A_{2}}\|g\|_{L^{2}\left(w^{-1}\right)}^{2} .
$$

Let $g^{*}=g w^{-1}$. Then this becomes

$$
\sum_{I \in D}\left\langle g^{*}, \frac{h_{I}}{\left(w_{I}^{-1}\right)^{1 / 2}}\right\rangle_{d x}^{2} \leq\|w\|_{A_{2}}\left\|g^{*}\right\|_{L^{2}(w)}^{2} .
$$

The left hand side can be rewritten as

$$
\sum_{I \in D}\left\langle g^{*}, h_{I}\left(w_{I}\right)^{1 / 2} w w^{-1}\right\rangle_{d x}^{2} \frac{1}{\mu(I)} .
$$

So finally, we have the inequality

$$
\sum_{I \in D}\left\langle g^{*}, g_{I}\right\rangle_{w}^{2} \leq\|w\|_{A_{2}}^{2}\left\|g^{*}\right\|_{L^{2}(w)}^{2}
$$

which is what we needed to prove.

Remark 4.5. We have the following inequality:

$$
\frac{1}{|J|} \sum_{I \subset J}\left(w_{I}^{-1}\right)^{2} d_{I}^{2} w_{I} \leq c\|w\|_{A_{2}}^{2} w_{J}^{-1}
$$

This inequality follows by realizing that the left hand side is bounded by $\left\|S\left(w^{-1} \chi_{J}\right)\right\|_{L^{2}(w)}^{2}$, which by Theorem 4.4 is bounded by $c\|w\|_{A_{2}}^{2}\left\|w^{-1} \chi_{J}\right\|_{L^{2}(w)}^{2}$. 


\section{Lemma 4.6.}

$$
\sum_{I \subset J}\left(\frac{c_{I}}{\sqrt{|I|}}\right)^{2} w_{I}|I| \leq c\|w\|_{A_{2}} w_{J}|J|,
$$

and this estimate is sharp.

Proof. By [1], 2.2 we have the following two estimates:

$$
\sum_{I \subset J}\left(w_{I}\right)^{p}\left(\frac{w_{I_{l}}-w_{I_{r}}}{w_{I}}\right)^{2}|I| \leq \frac{c}{p(p-1)}\|w\|_{B_{p}}^{p}\left(w_{J}\right)^{p}|J|,
$$

for $w \in B_{p}, p>1$, and

$$
\sum_{I \subset J}\left(w_{I}\right)^{q}\left(\frac{w_{I_{l}}-w_{I_{r}}}{w_{I}}\right)^{2}|I| \leq \frac{c}{q(1-q)}\left(w_{J}\right)^{q}|J|,
$$

for $0<q<1$. ( $c$ in both estimates is an absolute constant.)

Using Hölder's inequality on $\left(w_{I}\right)^{(1+\epsilon) / 2}$ and $\left(w_{I}\right)^{(1-\epsilon) / 2}$ we get

$$
\sum_{I \subset J}\left(\frac{c_{I}}{\sqrt{|I|}}\right)^{2} w_{I}|I| \leq\left(\sum_{I \subset J}\left(\frac{c_{I}}{\sqrt{|I|}}\right)^{2}\left(w_{I}\right)^{1+\epsilon}|I|\right)^{1 / 2}\left(\sum_{I \subset J}\left(\frac{c_{I}}{\sqrt{|I|}}\right)^{2}\left(w_{I}\right)^{1-\epsilon}|I|\right)^{1 / 2}
$$

where we choose $\epsilon$ to be $c /\|w\|_{A_{2}}$ for c a small constant.

If $\mathrm{c}$ is chosen smaller than a dimensional constant, this $\epsilon$ is one for which the reverse Hölder inequality holds for $w$, i.e., $\left(\frac{1}{|I|} \int_{I} w^{1+\epsilon}\right)^{\frac{1}{1+\epsilon}} \leq 2 \frac{1}{|I|} \int_{I} w$ as can be seen by carefully reading the proof of the reverse Hölder inequality in [2]. Therefore $w$ is in $B_{1+\epsilon}$ with norm 2 .

Letting $p=1+\epsilon$ and $q=1-\epsilon$ in the above estimates, and writing $\frac{w_{I_{l}}-w_{I_{r}}}{2 w_{I}} \sqrt{|I|}$ as $c_{I}$ we get

$$
\left(\sum_{I \subset J}\left(\frac{c_{I}}{\sqrt{|I|}}\right)^{2}\left(w_{I}\right)^{1+\epsilon}|I|\right)^{1 / 2} \leq\left(\frac{c}{\epsilon(1+\epsilon)}\right)^{1 / 2} 2^{\frac{1+\epsilon}{2}}\left(w_{J}\right)^{\frac{1+\epsilon}{2}}|J|^{1 / 2}
$$

and

$$
\left(\sum_{I \subset J}\left(\frac{c_{I}}{\sqrt{|I|}}\right)^{2}\left(w_{I}\right)^{1-\epsilon}|I|\right)^{1 / 2} \leq\left(\frac{c}{\epsilon(1-\epsilon)}\right)^{1 / 2}\left(w_{J}\right)^{\frac{1-\epsilon}{2}}|J|^{1 / 2}
$$

which, when combined, yields the required estimate. (Remember that $\epsilon=$ $\left.c /\|w\|_{A_{2}} \cdot\right)$

To see that this estimate is sharp, let $w=\prod_{i \in \mathbb{N}}\left(1+a 2^{-i / 2} h_{I_{i}}\right)$ where $h_{I_{i}}$ is the Haar function whose support is of the form $\left[0,2^{-i}\right]$. Then $\|w\|_{A_{2}}$ is comparable to $\frac{1}{1-a}$, and $\sum_{I \subset J}\left(\frac{c_{I}}{\sqrt{|I|}}\right)^{2} w_{I}|I|=\sum_{i \geq j} a^{2}\left(\frac{1+a}{2}\right)^{i}$.

Lemma 4.7. $\sum_{K \subset I} \frac{\left|c_{K}\right|}{\sqrt{|K|}} \frac{\left|d_{K}\right|}{\sqrt{|K|}} \mu(K)|K| \leq C\|w\|_{A_{2}}|I|$. 
Proof. By Lemma 5.3 from [6], we have

$$
\frac{1}{|J|} \sum_{I \subset J} v_{I} w_{I}\left|\frac{v_{I_{l}}-v_{I_{r}}}{v_{I}} \frac{w_{I_{l}}-w_{I_{r}}}{w_{I}}\right||I| \leq C \sqrt{v_{J} w_{J}},
$$

provided $v_{I} w_{I} \leq 1$ for all $I \subset J$.

Letting $v_{I}=\frac{w_{I}^{-1}}{\|w\|_{A_{2}}}$, and clearing the denominators, we get the above lemma.

Lemma 4.8. $\frac{1}{|J|} \sum_{I \subset J} \frac{\left|c_{I}\right|}{\sqrt{|I|}} \frac{\left|d_{I}\right|}{\sqrt{|I|}} w_{I}|I| \leq C\|w\|_{A_{2}} w_{J}$.

Proof. This lemma will be proven by the method of Bellman functions.

Let $B(x, y)=x\left(\frac{-4 A}{x y}-\frac{x y}{4 A}+4 A+1\right)$. Then $B(x, y)$ has the following properties, which can be checked by elementary calculus:

1. $0 \leq B(x, y) \leq 5 A x$ on $\{x, y>0 ; 1 \leq x y \leq A\}$

2. $\left(\begin{array}{cc}-B_{x x} & -B_{x y}-\frac{1}{y} \\ -B_{x y}-\frac{1}{y} & -B_{y y}\end{array}\right)$ is positive semidefinite on $\{x, y>0 ; 1 \leq x y \leq$ $2 A\}$.

3. $\left(\begin{array}{cc}-B_{x x} & -B_{x y}+\frac{1}{y} \\ -B_{x y}+\frac{1}{y} & -B_{y y}\end{array}\right)$ is positive semidefinite on $\{x, y>0 ; 1 \leq x y \leq$ $2 A\}$.

Let us show that these conditions imply the following discrete condition:

$$
B(x, y)-\frac{B\left(x_{-}, y_{-}\right)+B\left(x_{+}, y_{+}\right)}{2} \geq C\left|\left(x_{-}-x_{+}\right)\left(y_{-}-y_{+}\right) \frac{1}{y}\right|,
$$

where $x=\frac{\left(x_{-}+x_{+}\right)}{2}$ and $y=\frac{\left(y_{-}+y_{+}\right)}{2}$, and $(x, y),\left(x_{-}, y_{-}\right),\left(x_{+}, y_{+}\right)$are in the domain $\{x, y>0 ; 1 \leq x y \leq A\}$. Let $x(t)=\frac{x_{-}(1+t)+x_{+}(1-t)}{2}$ and $y(t)=$ $\frac{y_{-}(1+t)+y_{+}(1-t)}{2}$ for $t \in[-1,1]$. Note that $y(t) \leq \frac{2 y_{-}+2 y_{+}}{2}=2 y$. Denote by $b(t)$ the function $B(x(t), y(t))$. Note that $(x(t), y(t))$ are in the domain $\{x, y>0 ; 1 \leq x y \leq 2 A\}$. Then

$$
\begin{aligned}
-b^{\prime \prime}(t) & =\left(x^{\prime}(t), y^{\prime}(t)\right)\left(-d^{2} B\right)\left(x^{\prime}(t), y^{\prime}(t)\right)^{t} \\
& \geq\left(x^{\prime}(t), y^{\prime}(t)\right)\left(\begin{array}{cc}
0 & 1 / y(t) \\
1 / y(t) & 0
\end{array}\right)\left(x^{\prime}(t), y^{\prime}(t)\right)^{t},
\end{aligned}
$$

and

$$
\begin{aligned}
-b^{\prime \prime}(t) & =\left(x^{\prime}(t), y^{\prime}(t)\right)\left(-d^{2} B\right)\left(x^{\prime}(t), y^{\prime}(t)\right)^{t} \\
& \geq\left(x^{\prime}(t), y^{\prime}(t)\right)\left(\begin{array}{cc}
0 & -1 / y(t) \\
-1 / y(t) & 0
\end{array}\right)\left(x^{\prime}(t), y^{\prime}(t)\right)^{t} .
\end{aligned}
$$

Therefore, evaluating $x^{\prime}(t)$ and $y^{\prime}(t)$ we have

$$
-b^{\prime \prime}(t) \geq \frac{2}{y(t)}\left|\frac{\left(x_{-}-x_{+}\right)}{2} \frac{\left(y_{-}-y_{+}\right)}{2}\right| \geq \frac{1}{4 y}\left|\left(x_{-}-x_{+}\right)\left(y_{-}-y_{+}\right)\right| \text {. }
$$


By the definition of $b$,

$$
\begin{aligned}
& B(x, y)-\frac{B\left(x_{-}, y_{-}\right)+B\left(x_{+}, y_{+}\right)}{2}=b(0)-\frac{b(1)+b(-1)}{2} \\
&=-1 / 2 \int_{-1}^{1} b^{\prime \prime}(t)(1-|t|) d t \geq C\left|\left(x_{-}-x_{+}\right)\left(y_{-}-y_{+}\right) \frac{1}{y}\right| .
\end{aligned}
$$

Now we are ready to run the usual Bellman function argument to prove the lemma.

Let $x=w_{J}, y=w_{J}^{-1}, x_{-}=w_{J_{l}}, x_{+}=w_{J_{r}}, y_{-}=w_{J_{l}}^{-1}, y_{+}=w_{J_{r}}^{-1}$ and $A=$ $\|w\|_{A_{2}}$. These $x, y, x_{+}$, etc. satisfy the conditions for $(*)$. Therefore, we have

$$
c\left|w_{J_{l}}-w_{J_{r}}\right|\left|w_{J_{l}}^{-1}-w_{J_{r}}^{-1}\right| \frac{1}{w_{J}^{-1}}+\frac{B\left(w_{J_{l}}, w_{J_{l}}^{-1}\right)+B\left(w_{J_{r}}, w_{J_{r}}^{-1}\right)}{2} \leq B\left(w_{J}, w_{J}^{-1}\right) .
$$

Now $x_{-} y_{-}$and $x_{+} y_{+} \leq A=\|w\|_{A_{2}}$ again, so we can use estimate $(*)$ on the Bellman functions on the left side, too. This process can be repeated as often as we want. After $n$ iterations, we have the following formula:

$c \sum_{I \subset J,|I| \geq 2^{-n}|J|} \frac{\left|c_{I}\right|}{\sqrt{|I|}} \frac{\left|d_{I}\right|}{\sqrt{|I|}} w_{I} \frac{|I|}{|J|}+$ positive terms $\leq B\left(w_{J}, w_{J}^{-1}\right) \leq 5\|w\|_{A_{2}} w_{J}$.

So letting $n$ go to $\infty$, we get the desired estimate.

\section{Dividing the estimate up into 4 sums}

To begin with, let us estimate the norm of the martingale transform by duality,

$$
\begin{aligned}
\left\|T_{r} f\right\|_{L^{2}(w)} & =\sup _{\|g\|_{L^{2}\left(w^{-1}\right)}=1} \int T_{r} f g d x \\
& =\sup _{\|g\|_{L^{2}\left(w^{-1}\right)}=1} \int \sum_{I, J} r(I)\left\langle f, h_{I}\right\rangle h_{I}\left\langle g, h_{J}\right\rangle h_{J} d x .
\end{aligned}
$$

Since the $h_{I}$ 's are orthonormal in $L^{2}(d x)$ this becomes

$$
\sup _{\|g\|_{L^{2}\left(w^{-1}\right)}=1} \sum_{I} r(I)\left\langle f, h_{I}\right\rangle\left\langle g, h_{I}\right\rangle .
$$

Replacing $f$ by $f w^{1 / 2}$, and $g$ by $g w^{-1 / 2}$ this becomes:

$$
\left\|T_{r}\right\|_{L^{2}(w) \rightarrow L^{2}(w)}=\sup _{\|g\|_{L^{2}(d x)}=1\|f\|_{L^{2}(d x)}=1} \sum_{I} r(I)\left\langle f w^{-1 / 2}, h_{I}\right\rangle\left\langle g w^{1 / 2}, h_{I}\right\rangle .
$$

In order to estimate this, it is convenient to express the Haar functions in terms of a different family, which is more suited to working with weights.

The following "Haar functions" for $L^{2}(w)$ are normalized and orthogonal in $L^{2}(w)$ :

$$
h_{I}^{w}(x)=\frac{h_{I}(x)+\gamma_{w}^{I} \chi_{I}}{\delta_{w}^{I}}
$$


where $\gamma_{w}^{I}=\frac{-c_{I}}{|I|}$ and $\delta_{w}^{I}{ }^{2}=w_{I}\left(1-c_{I}^{2} /|I|\right)=\frac{w_{I_{l}} w_{I_{r}}}{w_{I}}$ are chosen to make these functions an orthonormal family.

Of course, there is the equivalent family for $w^{-1}$, with $\gamma_{w^{-1}}^{I}=\frac{-d_{I}}{|I|}$ and $\delta_{w^{-1}}^{I}{ }^{2}=w_{I}^{-1}\left(1-d_{I}^{2} /|I|\right)=\frac{w_{I_{l}}^{-1} w_{I_{r}}^{-1}}{w_{I}^{-1}}$.

Substituting $h_{I}(x)=\delta_{w}^{I} h_{I}^{w}(x)-\gamma_{w}^{I} \chi_{I}$ (or the equivalent for $w^{-1}$ ) into our equation, and sorting the different types of terms, we can write what we are trying to estimate as four sums $I+I I+I I I+I V$

$$
\begin{gathered}
I: \sum_{I \in D[0,1]} r(I)\left\langle f w^{-1 / 2}, h_{I}^{w^{-1}}\right\rangle \delta_{w^{-1}}^{I}\left\langle g w^{1 / 2}, h_{I}^{w}\right\rangle \delta_{w}^{I}, \\
I I:-\sum_{I \in D[0,1]} r(I)\left\langle f w^{-1 / 2}, \chi_{I}\right\rangle \gamma_{w^{-1}}^{I}\left\langle g w^{1 / 2}, h_{I}^{w}\right\rangle \delta_{w}^{I}, \\
I I I:-\sum_{I \in D[0,1]} r(I)\left\langle f w^{-1 / 2}, h_{I}^{w^{-1}}\right\rangle \delta_{w^{-1}}^{I}\left\langle g w^{1 / 2}, \chi_{I}\right\rangle \gamma_{w}^{I}, \\
I V: \sum_{I \in D[0,1]} r(I)\left\langle f w^{-1 / 2}, \chi_{I}\right\rangle \gamma_{w^{-1}}^{I}\left\langle g w^{1 / 2}, \chi_{I}\right\rangle \gamma_{w}^{I} .
\end{gathered}
$$

We will estimate each sum separately in absolute value.

5.1. Sum I. Since $r(I)$ could be any combination of signs, we will sum in absolute value.

$$
I: \sum\left|\left\langle f w^{-1 / 2}, h_{I}^{w^{-1}}\right\rangle\left\langle g w^{1 / 2}, h_{I}^{w}\right\rangle\right| \sqrt{\frac{w_{I_{l}} w_{I_{r}} w_{I_{l}}^{-1} w_{I_{r}}^{-1}}{w_{I} w_{I}^{-1}}} .
$$

Since $w_{I_{r}} w_{I_{l}} \leq\left(w_{I}\right)^{2}$ and $w_{I_{r}}^{-1} w_{I_{l}}^{-1} \leq\left(w_{I}^{-1}\right)^{2}$, this is bounded by

$$
\begin{aligned}
\sum\left|\left\langle f w^{-1 / 2}, h_{I}^{w^{-1}}\right\rangle\left\langle g w^{1 / 2}, h_{I}^{w}\right\rangle\right| & \sqrt{\mu(I)} \\
& \leq\|w\|^{1 / 2} \sum\left|\left\langle f w^{-1 / 2}, h_{I}^{w^{-1}}\right\rangle\left\langle g w^{1 / 2}, h_{I}^{w}\right\rangle\right| .
\end{aligned}
$$

Taking the inner product in $L^{2}(w)$ and $L^{2}\left(w^{-1}\right)$ instead of in $L^{2}(d x)$, this becomes

$$
\|w\|^{1 / 2} \sum\left|\left\langle f w^{1 / 2}, h_{I}^{w^{-1}}\right\rangle_{w^{-1}}\left\langle g w^{-1 / 2}, h_{I}^{w}\right\rangle_{w}\right|
$$

Since $h^{w}$ and $h^{w^{-1}}$ are orthonormal in $L^{2}(w)$ and $L^{2}\left(w^{-1}\right)$, Cauchy Schwarz and the Bessel inequality allow us to estimate this by

$$
\|w\|^{1 / 2}\left\|f w^{1 / 2}\right\|_{L^{2}\left(w^{-1}\right)}\left\|g w^{-1 / 2}\right\|_{L^{2}(w)}=\|w\|^{1 / 2}\|f\|_{L^{2}(d x)}\|g\|_{L^{2}(d x)} .
$$


5.2. Sum II and Sum III. Sums II and III are equivalent, so we will show Sum II only.

$$
\begin{aligned}
\sum_{I \in D[0,1]} \mid\left\langle f w^{-1 / 2},\right. & \left.\chi_{I}\right\rangle \gamma_{w^{-1}}^{I}\left\langle g w^{1 / 2}, h_{I}^{w}\right\rangle \delta_{w}^{I} \mid \\
& =\sum_{I \in D[0,1]}\left|\left(f w^{-1 / 2}\right)_{I}\right| I\left|\frac{\left|d_{I}\right|}{|I|}\left\langle g w^{1 / 2}, h_{I}^{w}\right\rangle \sqrt{w_{I}\left(1-c_{I}^{2} /|I|\right)}\right| .
\end{aligned}
$$

Using the fact that $\left(1-c_{I}^{2} /|I|\right) \leq 1$ and applying the Cauchy-Schwarz inequality, this becomes

$$
\left(\sum_{I \in D[0,1]}\left|\left(f w^{-1 / 2}\right)_{I}^{2}\right|\left|d_{I}\right|^{2} w_{I}\right)^{1 / 2}\left(\sum_{I \in D[0,1]}\left\langle g w^{1 / 2}, h_{I}^{w}\right\rangle^{2}\right)^{1 / 2}
$$

The second of these terms can again be estimated as in Sum I, and is bounded by $\|g\|_{L^{2}(d x)}$. To estimate the first term, we will use Theorem 4.1 with $w^{-1}$ for $w$, and $\alpha_{I}=d_{I}^{2} w_{I}$, and the inequality from Remark 4.5:

$$
\frac{1}{|J|} \sum_{I \subset J}\left(w_{I}^{-1}\right)^{2} d_{I}^{2} w_{I} \leq c\|w\|_{A_{2}}^{2} w_{J}^{-1}
$$

Theorem 4.1 gives us

$$
\left(\sum_{I \in D[0,1]}\left|\left(f w^{-1 / 2}\right)_{I}^{2}\right|\left|d_{I}\right|^{2} w_{I}\right)^{1 / 2} \leq c\|w\|_{A_{2}}\|f\|_{L^{2}(d x)} .
$$

Therefore Sum II is bounded by $c\|w\|_{A_{2}}\|f\|_{L^{2}(d x)}\|g\|_{L^{2}(d x)}$.

\subsection{Sum IV.}

$$
I V: \sum_{I \in D[0,1]}\left|\left(f w^{-1 / 2}\right)_{I}\right| I\left|\left(g w^{1 / 2}\right)_{I}\right| I\left|\frac{\left|c_{I} d_{I}\right|}{|I|^{2}}\right|
$$

In order to estimate this sum, we will make use of Theorem 4.2. with $\alpha_{I}=\frac{\left|c_{I} d_{I}\right|}{|I|}$. Since $w$ and $w^{-1}$ have the same $A_{2}$ norm, it is sufficient to calculate one of the conditions for the theorem.

$$
\begin{aligned}
\frac{1}{|J|} \int_{J}\left(\sum_{I \subset J} \alpha_{I} w_{I} \chi_{I}(x)\right)^{2} & w^{-1} d x \\
& =\frac{1}{|J|} \sum_{I, K \subset J} \alpha_{I} w_{I} \alpha_{K} w_{K} \int_{J} \chi_{I}(x) \chi_{K}(x) w^{-1}(x) d x .
\end{aligned}
$$


We can break this sum into the case where $I=K$ or $I \subset K$, since $K \subset I$ is equivalent to the latter. So we get

$$
\frac{1}{|J|}\left(\sum_{I \subset J} \alpha_{I}^{2}\left(w_{I}\right)^{2} w_{I}^{-1}|I|+2 \sum_{I, K K \subset I \subset J} \alpha_{I} \alpha_{K} w_{K} w_{I} w_{K}^{-1}|K|\right) .
$$

The first sum is

$$
\frac{1}{|J|} \sum_{I \subset J}\left(\frac{c_{I}}{\sqrt{|I|}}\right)^{2}\left(\frac{d_{I}}{\sqrt{|I|}}\right)^{2} w_{I}|I| \mu(I) .
$$

Because $w$ is an $A_{\infty}$ weight, $\left|\frac{d_{I}}{\sqrt{|I|}}\right| \leq 1$ (see [4]), and so we can estimate the above by

$$
\frac{1}{|J|} \sum_{I \subset J}\left(\frac{c_{I}}{\sqrt{|I|}}\right)^{2} w_{I}|I| \mu(I)
$$

which is $\leq c\|w\|_{A_{2}}^{2} w_{J}$ by Remark 4.5 .

This leaves us to estimate the second sum

$$
\begin{aligned}
\frac{2}{|J|} \sum_{I, K K \subset I \subset J} \frac{\left|c_{I}\right|}{\sqrt{|I|}} \frac{\left|d_{I}\right|}{\sqrt{|I|}} & \frac{\left|c_{K}\right|}{\sqrt{|K|}} \frac{\left|d_{K}\right|}{\sqrt{|K|}} w_{I} \mu(K)|K| \\
& =\frac{2}{|J|} \sum_{I \subset J} \frac{\left|c_{I}\right|}{\sqrt{|I|}} \frac{\left|d_{I}\right|}{\sqrt{|I|}} w_{I} \sum_{K \subset I} \frac{\left|c_{K}\right|}{\sqrt{|K|}} \frac{\left|d_{K}\right|}{\sqrt{|K|}} \mu(K)|K| .
\end{aligned}
$$

The inside sum, by lemma 4.7 is bounded by $c\|w\|_{A_{2}}|I|$. That leaves us to estimate

$$
\|w\|_{A_{2}} \frac{2}{|J|} \sum_{I \subset J} \frac{\left|c_{I}\right|}{\sqrt{|I|}} \frac{\left|d_{I}\right|}{\sqrt{|I|}} w_{I}|I|
$$

which, by lemma 4.8 is bounded by $c\|w\|_{A_{2}}^{2} w_{J}$. Remembering that the final estimate for sum IV will be the square root of the estimate for the above, we have that sums I to IV are each individually bounded by $c\|w\|_{A_{2}}\|f\|_{L^{2}(d x)}\|g\|_{L^{2}(d x)}$, which concludes the proof.

\section{References}

[1] S.M. Buckley, Summation conditions on weights, Michigan Math. J. 40 (1993), 153-170.

[2] R.R. Coifman and C. Fefferman. Weighted norm inequalities for maximal functions and singular integrals, Studia Math. 51 (1974), 241-250.

[3] R. Fefferman and J. Pipher, Multiparameter operators and sharp weighted inequalities, Amer. J. Math. 119 (1997), 337-369.

[4] R.A. Fefferman, C. E. Kenig, and J. Pipher, The theory of weights and the Dirichlet problem for elliptic equations, Ann. of Math. (2) 134 (1991), 65-124.

[5] S. Hukovic, F. Nazarov, S. Treil, and A. Volberg, The Bellman functions and sharp weighted inequalities for square functions, preprint.

[6] F. Nazarov, S. Treil, and A. Volberg, The Bellman functions and two weight inequalities for Haar multipliers, preprint. 
[7] S. Treil and A. Volberg, Wavelets and the angle between past and future, J. Funct. Anal. 143 (1997), 269-308.

Department of Mathematics, University of Chicago, 5734 University Avenue, Chicago, Il 60637

E-mail address: jwittwer@math.uchicago.edu 